\title{
Long Noncoding RNA ANROC on the INK4 Locus Functions to Suppress Cell Proliferation
}

\author{
YOJIRO KOTAKE ${ }^{1,2}$ and TAKESHI TSURUDA ${ }^{2}$ \\ ${ }^{1}$ Graduate School of Humanity-Oriented Science and Engineering, Kindai University, Fukuoka, Japan; \\ ${ }^{2}$ Department of Biological and Environmental Chemistry, \\ Faculty of Humanity-Oriented Science and Engineering, Kindai University, Fukuoka, Japan
}

\begin{abstract}
Background/Aim: The INK4 locus encodes three important genes p15 INK4B, p16 INK4A, and ARF, which function to suppress oncogenesis, and a long noncoding RNA, ANRIL, which, in contrast, functions to promote oncogenesis. Herein, we report a fifth genetic element on the INK4 locus, a long noncoding RNA with unknown function named associated negative regulation of cell proliferation (ANROC), which played a role in the suppression of cell proliferation. Materials and Methods: Following ANROC silencing in cells by siRNA, quantitative reverse transcription-polymerase chain reaction ( $q R T-P C R)$ and cell cycle analysis using flow cytometry were performed. Results: ANROC expression was decreased by oncogenic RAS signalling. ANROC knockdown enhanced HeLa cell proliferation and induced cyclin BI mRNA, which promotes G2/M progression of the cell cycle. Furthermore, flow cytometric analysis revealed that ANROC knockdown increased the percentage of cells in the $S$ and $G_{2} / M$ phases of the cell cycle. Conclusion: ANROC functions to suppress cell cycle progression by suppressing cyclin BI expression, thus inhibiting cell proliferation.
\end{abstract}

The INK4 locus is located on human chromosome 9p21 and is mutated, deleted, and transcriptionally suppressed in a wide range of human cancers $(1,2)$. This locus encodes three tumour suppressor genes, the CDK inhibitors $p 16^{I N K 4 A}$ and $p 15^{I N K 4 B}$, and the p53 stabilizing factor $A R F$, which are mainly regulated at the transcriptional level (3). Along with Bracken et al., we

This article is freely accessible online.

Correspondence to: Yojiro Kotake, Ph.D., Department of Biological and Environmental Chemistry, Faculty of Humanity-Oriented Science and Engineering, Kindai University, 11-6 Kayanomori, Iizuka, Fukuoka 820-8555, Japan. Tel: +81 0948225659 (469), Fax: +810948230536, e-mail: ykotake@fuk.kindai.ac.jp

Key Words: INK4 locus, long noncoding RNA, cell cycle, cyclin B1, CDK inhibitor. have reported that polycomb repression complex 1 (PRC1) and 2 (PRC2) directly bind to and epigenetically suppress $p 16^{I N K 4 A}$ and $p 15^{I N K 4 B}$ transcription on the INK4 locus $(4,5)$. Furthermore, we and Yap et al. have revealed that an antisense noncoding RNA transcribed from the INK4 locus (ANRIL) binds to and recruits PRC1 and PRC2 to the INK4 locus, thus suppressing $p 16^{I N K 4 A}$ and $p 15^{I N K 4 B}$ transcription $(6,7)$. ANRIL is classified as a long noncoding RNA (lncRNA); these are defined by lengths exceeding 200 nucleotides. Recent studies have revealed that $\operatorname{lncRNAs}$ have various functions such as gene regulation, organization of nuclear architecture, and regulation of interacting proteins and RNAs, and are involved in normal physiology and disease (8). ANRIL also functions to suppress cellular senescence and to promote cancer cell proliferation, and thus is thought to be an oncogene $(6,7,9-11)$. Indeed, many studies of clinical cancer specimens have revealed that ANRIL is highly expressed in many types of human cancers such as gastric cancer, non-small cell lung cancer, hepatocellular carcinoma, ovarian cancer, and cervical cancer (12).

Cell proliferation is stringently modulated by the cell cycle, the progression of which is promoted by protein kinase complexes comprising cyclins and cyclin-dependent kinases (CDKs) and is negatively regulated by CDK inhibitors (CKIs) (13). Disruption of cell cycle regulation leads to oncogenic transformation (13). In this study, we identified another functional lncRNA transcribed from the INK4 locus named ANROC, and showed that it participates in the suppression of cell cycle progression via inhibiting cyclin B1, which leads to inhibition of cell proliferation.

\section{Materials and Methods}

Cell culture and small interfering RNA (siRNA). The Human cervical cancer cells HeLa were cultured as described previously (10). HeLa cells were infected with retroviruses carrying HRASG12V. Retrovirus generation and infection were performed as described previously (5). siRNAs were used to down-regulate ANROC expression in $\mathrm{HeLa}$ cells. ANROC siRNAs were synthesized by GeneDesign, Inc. (Osaka, Japan). The nucleotide sequence of ANROC siRNA was 5'ACCGCAUUUCAUCGAUCUU-3' with 3'dTdT overhangs. A total 


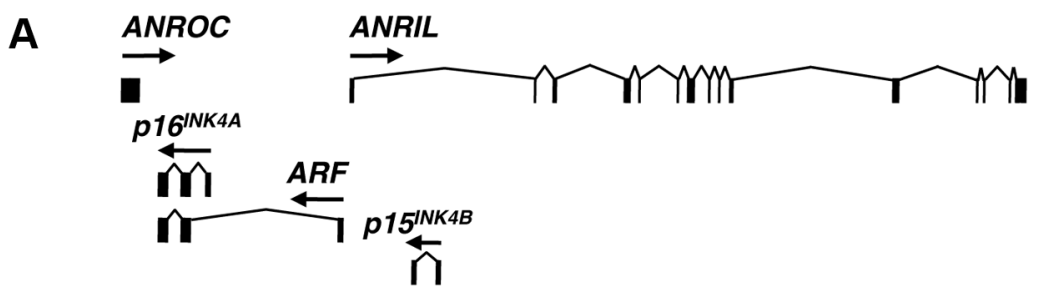

B

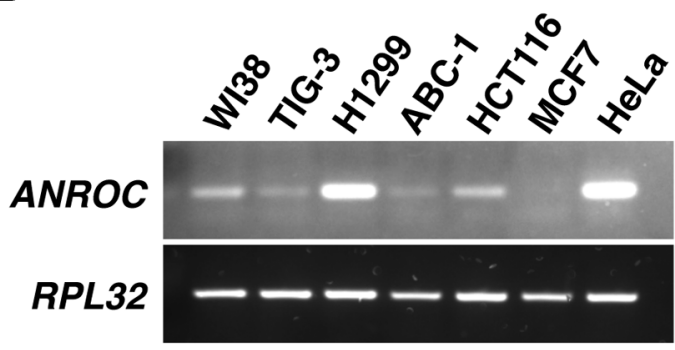

C

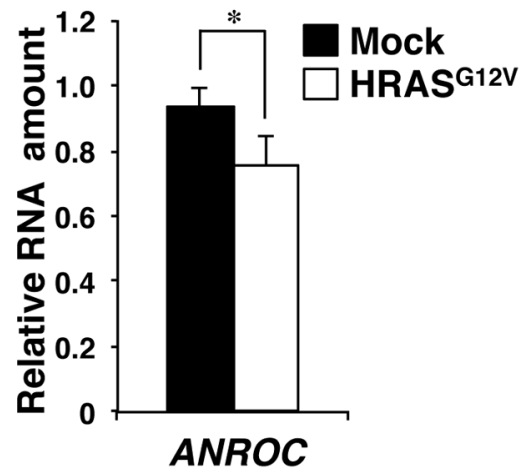

Figure 1. Analysis of ANROC expression. A: A schematic diagram of ANROC, ANRIL, p16INK4A, p15INK4B, and ARF. The arrow heads indicate the direction of transcription. The black boxes show the exons of each gene. B: ANROC expression in the indicated human cell lines was detected by RT-PCR. Ribosomal protein L32 (RPL32) expression was detected as an internal control. C: HeLa cells were infected with retroviruses carrying $H R A S^{G 12 V}$ or mock vector. The expression levels of ANROC were assessed by qRT-PCR. The results are shown as relative values based on the values of HeLa cells/mock. ${ }^{*} p<0.05$.

of $5 \times 10^{5} \mathrm{HeLa}$ cells were seeded and cultured for $24 \mathrm{~h}$, and then siRNA transfection was performed using Lipofectamine RNAiMAX (Thermo Fisher Scientific, Waltham, MA, USA) according to the manufacturer's instructions. Cells transfected with siRNAs for $72 \mathrm{~h}$ were subjected to quantitative reverse transcription-polymerase chain reaction (qRT-PCR) and cell cycle analysis.

$q R T-P C R$. Total RNA was extracted from cells using a RNeasy Plus kit (Qiagen, Hilden, Germany) and subjected to DNase treatment with a TURBO DNA-free Kit (Thermo Fisher Scientific) according to the manufacturer's instructions. cDNA synthesis was performed with the SuperScript III First-Strand Synthesis System (Thermo Fisher Scientific). The synthesized cDNAs were amplified using a QuantiTect SYBR Green PCR kit (Qiagen) with $200 \mathrm{nM}$ primers. The nucleotide sequences of primers were as follows: ANROC 5'CTGAGGCCTGGTGAGCAAAA-3' and 5'-AGACTCGAGACT GGCACATC-3', cyclin Al 5'-GCACACTCAAGTCAGACCTGCA3' and 5'-ATCACATCTGTGCCAAGACTGGA-3', cyclin B1 5'GACCTGTGTCAGGCTTTCTCTG-3' and 5'-GGTATTTTGGTC TGACTGCTTGC-3', cyclin D1 5'-GAGCTGCTGCAAATG GAACTG-3' and 5'-AAAGAAAGTGCGTTGTGCGG-3', cyclin E1 5'-TGTGTCCTGGATGTTGACTGCC-3' and 5'-CTCTA TGTCGCACCACTGATACC-3'. The nucleotide sequences of primers for $p 15^{I N K 4 B}, p 16^{I N K 4 A}, A R F, G A P D H$ (9), and ANRIL (10) have been described previously. Real-time PCR assays were performed using the Mx3000P Real-Time Q-PCR System (Agilent Technologies, Santa Clara, CA, USA).
Cell cycle analysis. A total of $1 \times 10^{6}$ cells were fixed with $70 \%$ ethanol overnight at $-20^{\circ} \mathrm{C}$ and stained using a Muse ${ }^{\mathrm{TM}}$ Cell Cycle Kit (Merck Millipore, Darmstadt, Germany) according to the manufacturer's instructions. The stained cells were then analysed with a Muse Cell Analyzer (Merck Millipore).

Statistical analysis. All experiments were performed in triplicate and the data are shown as means and standard deviations. The results were analysed using a two-tailed $t$-test using Microsoft Office Excel. $p$-Values of $<0.05$ were considered to indicate statistically significant differences.

\section{Results}

We have previously shown that the lncRNA ANRIL transcribed from the INK4 locus, suppressed $p 16^{I N K 4 A}$ and p $15^{I N K 4 B}$ transcription in a cis-acting manner (6). We also found another IncRNA with an unknown function (GebeBank ID: BX091351.1, CDKN2A divergent transcript) transcribed from the periphery of the INK4 locus by searching the expressed sequence tags (EST) database provided by the National Center for Biotechnology Information (NCBI; Figure 1A). In this study, we demonstrated that this IncRNA participates in the negative regulation of cell proliferation, and thus named it ANROC (associated negative regulation of 
A

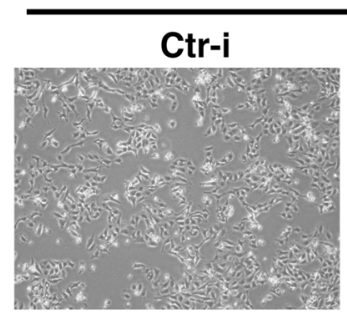

HeLa cells

B

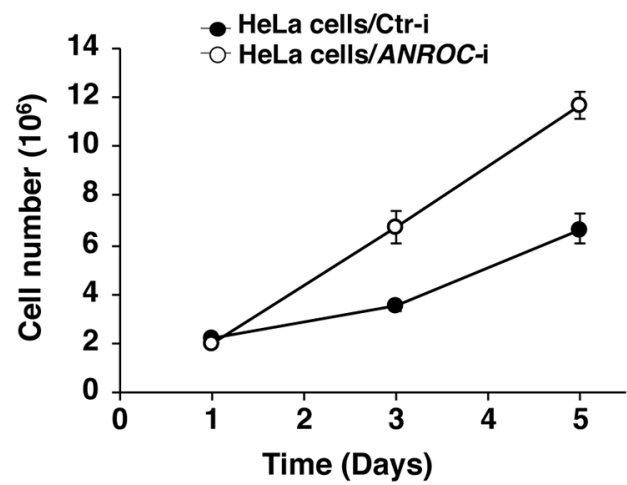

C

CAL27 cells/Ctr-i

CAL27 cells/ANROC-i

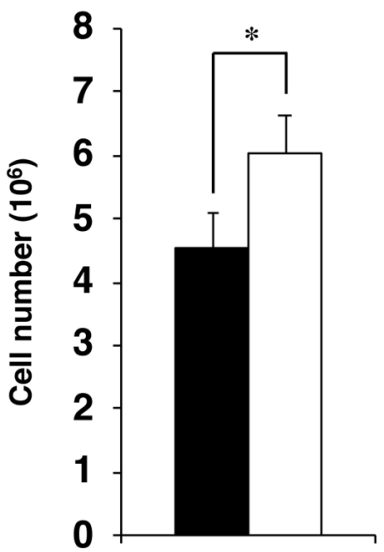

Figure 2. Silencing of ANROC promotes HeLa cell proliferation. A: Phase-contrast images of HeLa cells transfected with control siRNA (Ctr-i) or siRNA against ANROC (ANROC-i) for 72 h. B: Proliferation curves of HeLa cells/Ctr-i and /ANROC-i. Viable cells were counted by staining with trypan blue. The $x$-axis indicates days since transfection of siRNA. C: CAL27 cells transfected with siRNA for $72 h$ were counted by trypan blue staining. $* p<0.05$.

cell proliferation). The $A N R O C$ sequence comprises only one exon with a polyadenylation site, and the transcript length is 616 bases, but there is no significant open reading frame.

Initially, we analysed ANROC expression in WI38 and TIG-3 human normal diploid foetal lung fibroblasts and in H1299 and ABC-1 human non-small cell lung cancer, HCT116 human colorectal cancer, MCF7 human breast cancer, and HeLa human cervical cancer cells. RT-PCR data revealed that ANROC was highly expressed in H1299 and HeLa cells (Figure 1B). We have previously shown that oncogenic RAS signalling affects the transcription of the INK4 locus (6). The forced expression of oncogenic HRAS ${ }^{\mathrm{G} 12 \mathrm{~V}}$ induces $p 16^{I N K 4 A}$ and $p 15^{I N K 4 B}$ transcription and inversely suppresses $A N R I L$ expression (6). We, therefore, examined whether $A N R O C$ expression is also affected by oncogenic RAS signalling. qRT-PCR data revealed that ANROC RNA levels were decreased by overexpressing oncogenic HRAS ${ }^{\mathrm{G} 12 \mathrm{~V}}$, which indicates that ANROC expression is suppressed by oncogenic RAS signalling (Figure 1C).

We next examined the biological function of $A N R O C$ in HeLa cells, in which ANROC is highly expressed. Silencing of $A N R O C$ resulted in the promotion of $\mathrm{HeLa}$ cell proliferation (Figure $2 \mathrm{~A}$ and $\mathrm{B}$ ). The same result was obtained with the human oral cancer cell line CAL27 (Figure $2 \mathrm{C})$. These results suggested that ANROC functions to suppress cell proliferation.

Given that another lncRNA transcribed from the INK4 locus, ANRIL, suppresses $p 16^{I N K 4 A}$ and $p 15^{I N K 4 B}$ transcription (6), we examined whether ANROC also participates in the regulation of the INK4 locus. qRT-PCR data showed that $A N R O C$ knockdown caused an increase in p16 ${ }^{I N K 4 A}, p 15^{I N K 4 B}$, and ARF mRNA levels, but did not affect ANRIL RNA levels (Figure 3A), which suggests that $A N R O C$ plays a role in the suppression of $p 16^{I N K 4 A}$, $p 15^{I N K 4 B}$, and $A R F$ transcription. Even though the expression of $p 16^{I N K 4 A}, p 15^{I N K 4 B}$, and ARF was increased by ANROC knockdown, cell proliferation was unexpectedly promoted. We therefore examined whether ANROC regulates cyclins that promote cell proliferation. qRT-PCR data indicated that ANROC knockdown caused an increase in cyclin Bl mRNA levels (Figure 3B).

Given that ANROC knockdown increased cyclin Bl expression, which promotes transition to the G2/M of the cell cycle, we next examined whether ANROC participates in the regulation of the cell cycle. Cell cycle analysis showed that $A N R O C$ knockdown significantly decreased the proportion of cells in the $G_{1}$ phase and increased the number 

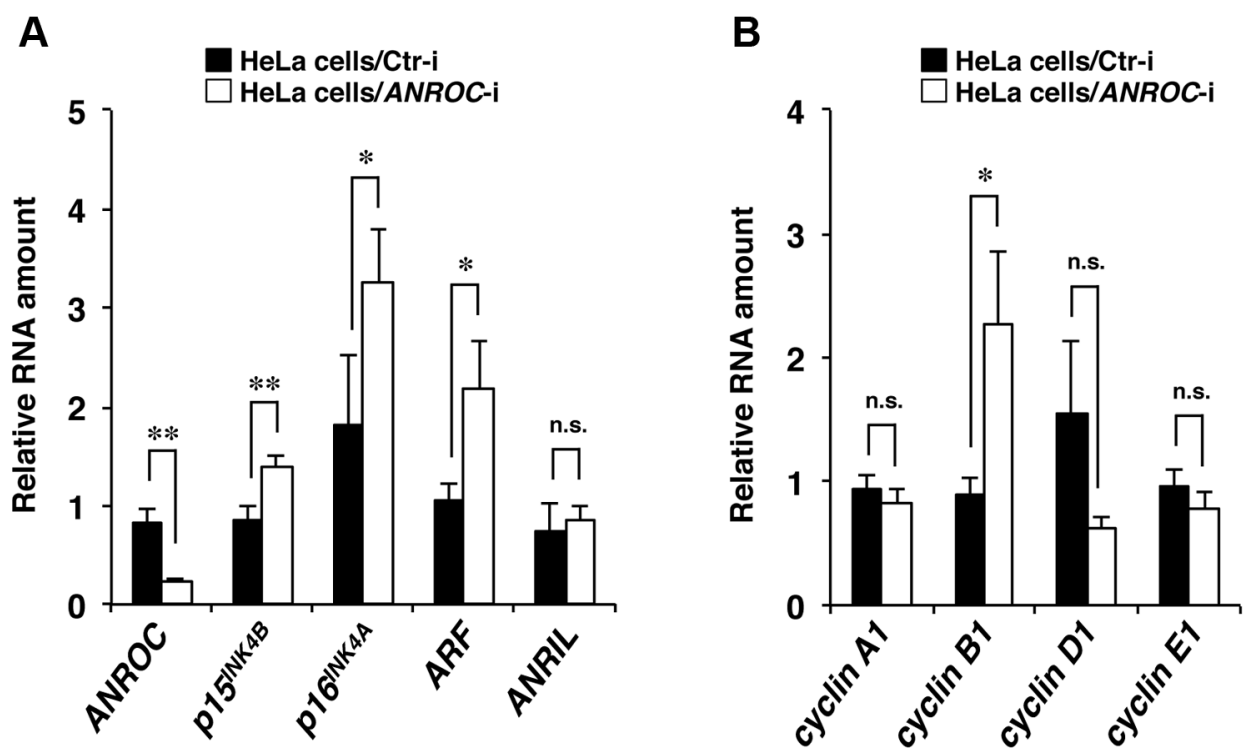

Figure 3. Silencing of ANROC increases the mRNA expression levels of p16INK4A, p15INK4B, ARF, and cyclin B1. The mRNA levels of ANROC, p16 INK4A, p15INK4B, ARF, ANRIL $(A)$ and cyclins $(B)$ were measured by qRT-PCR. The results are shown as relative values based on the values of HeLa cells transfected with control siRNA $\left(\right.$ Ctr-i). ${ }^{*} p<0.05, * * p<0.01$, n.s., not significant.

of cells in the $\mathrm{S}$ and $\mathrm{G}_{2} / \mathrm{M}$ phase (Figure $4 \mathrm{~A}$ and $\mathrm{B}$ ). Taken together, these data suggest that ANROC functions to suppress cell cycle progression via suppressing cyclin B1 expression.

\section{Discussion}

To date, many studies have revealed the biological importance of the INK4 locus, especially the function of the coding genes $p 16^{I N K 4 A}$ and $p 15^{I N K 4 B}$, and the stabilizing factor of p53 tumour suppressor $A R F$, as well as the long noncoding RNA ANRIL $(1,12)$. In this study, we identified a fifth functional genetic element of the INK4 locus, $A N R O C$, and revealed that it negatively regulates cell proliferation. We observed that ANROC expression is suppressed by oncogenic RAS signalling, which indicates that ANROC antagonizes the function of the oncogenic RAS signalling that causes hyperproliferation. In support of this, $A N R O C$ knockdown promoted the proliferation of HeLa and CAL27 cells, which suggests that ANROC functions to suppress cell proliferation. However, the molecular mechanism by which oncogenic RAS signalling suppresses $A N R O C$ expression is unclear and is an important issue that warrants further study.

We and Yap et al. have previously reported that the long noncoding RNA ANRIL suppresses the transcription of $p 16^{I N K 4 A}$ and $p 15^{I N K 4 B}$ on the INK4 locus in a cis-acting manner $(6,7)$. In this study, we also showed that silencing

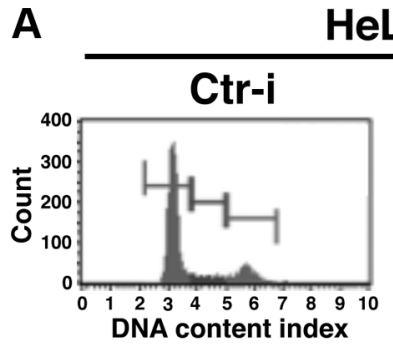

HeLa cells

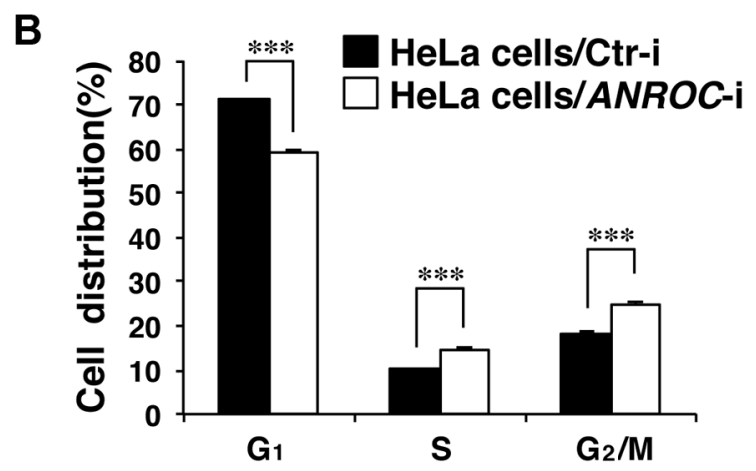

Figure 4. Silencing of ANROC results in an increase in the number of cells in the $S$ and $G_{2} / M$ phases and a decrease in the number of cells in the $G_{1}$ phase. A: Histogram of cell cycle analysis of HeLa cells transfected with control siRNA (Ctr-i) or siRNA against ANROC (ANROC-i) for $72 \mathrm{~h}$. The $x$-axes show the fluorescence intensity of propidium iodide (DNA content index). The y-axes show cell number. $B$ : The percentages of HeLa cells transfected with siRNA in the G1, S, and $G_{2} / M$ phase. *** $p<0.001$. 


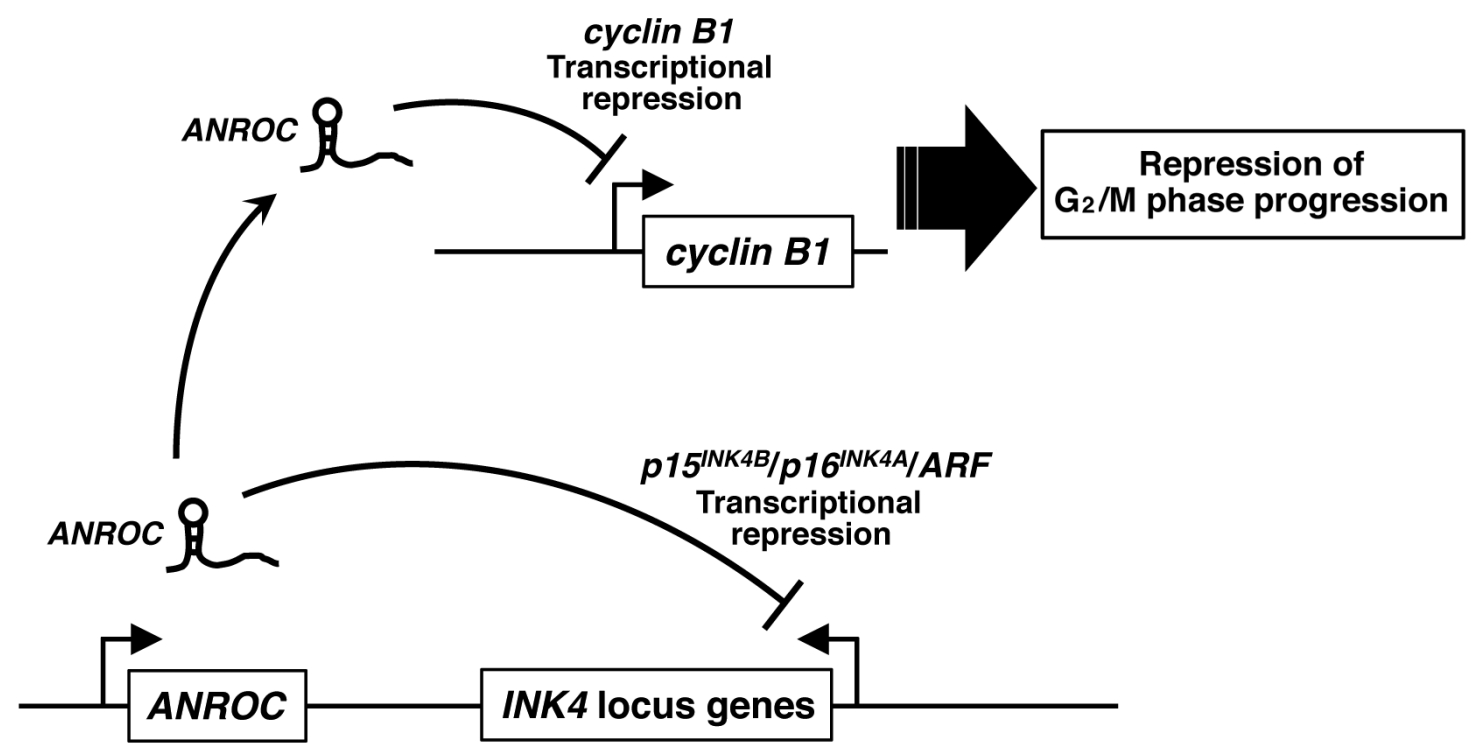

Figure 5. Model of ANROC function. ANROC transcribed from the INK4 locus suppresses the expression of p16INK4A, p15INK4B, ARF, and cyclin $B 1$ in cis- and trans-acting manners. ANROC suppresses the progression of the cell cycle, leading to the inhibition of cell proliferation.

of $A N R O C$ increased $p 16^{I N K 4 A}, p 15^{I N K 4 B}$, and $A R F$ expression, which implies that $A N R O C$ negatively regulates the transcription of the INK4 locus as well as ANRIL. Even though these negative regulators of cell proliferation were increased, silencing of ANROC promoted cell proliferation, which indicates that $A N R O C$ plays a role in the negative regulation of cell proliferation in a trans-acting manner. In support of this, ANROC knockdown increased cyclin $B I$ expression, promoting $\mathrm{G}_{2} / \mathrm{M}$ progression. We also showed that silencing of ANROC resulted in a decrease of cells in the $\mathrm{G}_{1}$ phase and an increase of cells in the $\mathrm{S}$ and $\mathrm{G}_{2} / \mathrm{M}$ phases. Taking together these results, $A N R O C$ is proposed to act as a negative regulator of cell cycle progression via suppressing cyclin $B 1$ expression, which leads to the inhibition of cell proliferation (Figure 5). Recent studies have shown that many lncRNAs function to regulate gene expression by associating with proteins such as polycomb proteins and transcription factors and miRNAs (8). The identification of factors associated with ANROC will be required to elucidate its mechanism of action.

The deletion, mutation, and transcriptional suppression of the INK4 locus is frequently observed in a wide range of human cancers (1). Thus, this locus is thought to be important in preventing oncogenesis. ANROC may also function as a tumour suppressor in addition to $p 16^{I N K 4 A}$ and $p 15^{I N K 4 B}$ on the INK4 locus. Clarification of the role of $A N R O C$ in oncogenesis is an important and requires further study.

\section{Conflicts of Interest}

The Authors have no conflicts of interest directly relevant to the contents of this article.

\section{Authors' Contributions}

Conceptualization and design, Y.K. and T.T.; supervision, Y.K.; materials, Y.K. and T.T.; data collection, Y.K. and T.T.; analysis, Y.K. and T.T.; and writing, Y.K.

\section{Acknowledgements}

The Authors thank Natsumi Matsunaga, Ryotaro Okada and the laboratory members for their technical assistance and discussions. This study was funded by JSPS KAKENHI Grant Number $17 \mathrm{~K} 07184$ (to YK), the Naito Foundation (to YK), and the Takeda Science Foundation (to YK). The Authors would also like to thank Nikki March, PhD, from Edanz Group (www.edanzediting.com/ac) for proofreading this manuscript.

\section{References}

1 Sharpless NE: INK4a/ARF: a multifunctional tumor suppressor locus. Mutat Res 576: 22-38, 2005. PMID: 15878778. DOI: 10.1016/j.mrfmmm.2004.08.021

2 Ortega S, Malumbres M, and Barbacid M: Cyclin D-dependent kinases, INK4 inhibitors and cancer. Biochim Biophys Acta 1602: 73-87, 2002. PMID: 11960696. DOI: 10.1016/s0304419x $(02) 00037-9$

3 Gil $\mathrm{J}$ and Peters G: Regulation of the INK4b-ARF-INK4a tumour suppressor locus: all for one or one for all. Nat Rev Mol 
Cell Biol 7: 667-677, 2006. PMID: 16921403. DOI: 10.1038/nrm1987

4 Bracken AP, Kleine-Kohlbrecher D, Dietrich N, Pasini D, Gargiulo G, Beekman C, Theilgaard-Monch K, Minucci S, Porse BT, Marine JC, Hansen KH and Helin K: The Polycomb group proteins bind throughout the INK4A-ARF locus and are disassociated in senescent cells. Genes Dev 21: 525-530, 2007. PMID: 17344414. DOI: 10.1101/gad.415507

5 Kotake Y, Cao R, Viatour P, Sage J, Zhang Y and Xiong Y: pRB family proteins are required for $\mathrm{H} 3 \mathrm{~K} 27$ trimethylation and Polycomb repression complexes binding to and silencing p16INK4alpha tumor suppressor gene. Genes Dev 21: 49-54, 2007. PMID: 17210787. DOI: $10.1101 / \mathrm{gad} .1499407$

6 Kotake Y, Nakagawa T, Kitagawa K, Suzuki S, Liu N, Kitagawa $\mathrm{M}$ and Xiong Y: Long non-coding RNA ANRIL is required for the PRC2 recruitment to and silencing of p15(INK4B) tumor suppressor gene. Oncogene 30: 1956-1962, 2011. PMID: 21151178. DOI: $10.1038 /$ onc.2010.568

7 Yap KL, Li S, Munoz-Cabello AM, Raguz S, Zeng L, Mujtaba S, Gil J, Walsh MJ and Zhou MM: Molecular interplay of the noncoding RNA ANRIL and methylated histone $\mathrm{H} 3$ lysine 27 by polycomb CBX7 in transcriptional silencing of INK4a. Mol Cell 38: 662-674, 2010. PMID: 21151178. DOI: 10.1038/onc.2010.568

8 Kopp F and Mendell JT: Functional Classification and experimental dissection of long noncoding RNAs. Cell 172: 393407, 2018. PMID: 29373828. DOI: 10.1016/j.cell.2018.01.011
9 Naemura M, Murasaki C, Inoue Y, Okamoto H and Kotake Y: Long noncoding RNA ANRIL regulates proliferation of non-small cell lung cancer and cervical cancer cells. Anticancer Res 35: 53775382, 2015. PMID: 29373828. DOI: 10.1016/j.cell.2018.01.011

10 Matsunaga N, Wakasaki T, Yasumatsu R and Kotake Y: Long noncoding RNA, ANRIL, regulates the proliferation of head and neck squamous cell carcinoma. Anticancer Res 39: 4073-4077, 2019. PMID: 31366490 . DOI: 10.21873/anticanres.13564

11 Zhang EB, Kong R, Yin DD, You LH, Sun M, Han L, Xu TP, Xia R, Yang JS, De W and Chen J: Long noncoding RNA ANRIL indicates a poor prognosis of gastric cancer and promotes tumor growth by epigenetically silencing of miR99a/miR-449a. Oncotarget 5: 2276-2292, 2014. PMID: 24810364. DOI: $10.18632 /$ oncotarget.1902

$12 \mathrm{Li} \mathrm{Z}$, Yu X and Shen J: ANRIL: a pivotal tumor suppressor long non-coding RNA in human cancers. Tumour Biol 37: 5657-5661, 2016. PMID: 26753962. DOI: 10.1007/s13277-016-4808-5

13 Sherr CJ: Cancer cell cycles. Science 274: 1672-1677, 1996. PMID: 8939849. DOI: 10.1126/science.274.5293.1672 